\title{
Energy Control for Complex Pendulums based on Tracking of Online Computed Force Trajectories*
}

\author{
Franz Christange $^{1}$, Philine Donner ${ }^{1,2}$ and Martin Buss ${ }^{1,2}$
}

\begin{abstract}
In this paper we propose to track online computed force trajectories to control the energy of various types of pendulum-like objects. The considered pendulum-like objects can be controlled by multiple agents and swing in different oscillation degrees of freedom. Our goal is to excite one specific oscillation, the intended oscillation, while damping all other disturbance oscillations. By approximating the intended oscillation as a simple pendulum oscillation, we can specify a desired force trajectory. Tracking of this force trajectory results in a controlled swing-up of the intended oscillation accompanied by a simultaneous damping of the disturbance oscillations. Simulation experiments with a two-agent trapezoidal pendulum show convincing control performance. A human-robot virtual reality experiment shows the transferability of the control approach to a human interaction partner. The limitations of the approach are discussed based on simulation results obtained for a single-agent double pendulum.
\end{abstract}

\section{INTRODUCTION}

This paper investigates energy control of pendulum-like objects under the influence of gravity. The most simple example for such a pendulum-like object is the simple pendulum. The theoretical background of simple pendulums, especially its nonlinear frequency characteristic, is well investigated, exemplary in [1]. Simple pendulums have been successfully used to describe various complex mechanisms like robotic walking [2], [3], dance partners [4] or fluid in containers [5]. A further example is a two link robot brachiating with pendulum-like dynamics [6].

In many applications, the goal is to damp oscillations, e.g. for cranes transporting goods [7] or for quadrotors with suspended load [8]. In [9], a suspended load is carried by two agents with the goal to damp residual oscillations resulting from high speed transport.

The controlled swing-up of pendulum-like objects has found a lot of attention in terms of swinging up simple, double or even higher order pendulums with the final goal of stabilizing them in their unstable equilibrium point e.g. [10][14]. The swing-up of a cable driven mechanism to a specific energy level by two coupled actuators is described in [15]. To the best of the authors' knowledge the swing-up of multiagent pendulums has hardly been discussed. Controlling the

\footnotetext{
*This work was supported in part within the ERC Advanced Grant SHRINE Agreement No. 267877 (www.shrine-project.eu) and in part by the Technische Universität München - Institute for Advanced Study (www.tumias.de), funded by the German Excellence Initiative

${ }^{1}$ Chair of Automatic Control Engineering, Technische Universität München, Theresienstr. 90, 80333 München, Germany, \{franz.eutermoser, philine.donner, mb\}@tum.de

${ }^{2}$ TUM Institute for Advanced Study, Technische Universität München, Lichtenbergstrasse 2a, 85748 Garching, Germany
}

trajectories of slung-loads carried by multiple helicopters [16] is related to this subject.

In our previous works [17], [18], human-robot object swinging is motivated by the extension of the manipulation capabilities with respect to bulky, flexible objects. Our exemplary task is cooperative swinging of a sports mat in order to lift it onto a trolley. As a first step towards human-robot dynamic object manipulation, the swing-up of pendulumlike objects, in [17] a v-shaped pendulum and in [18] a trapezoidal pendulum, is investigated.

In this paper, we present an energy control approach for complex pendulum-like objects based on force trajectory tracking. Similar to [18], the intended oscillation, the oscillation degree of freedom to be swung-up, is approximated by an abstract simple pendulum. In contrast to [18], the force based approach allows to simultaneously damp disturbance oscillations, without the need of an additional damping controller. The generalizability of the force based approach is discussed with respect to the simple pendulum approximation and by applying it not only to the two-agent trapezoidal pendulum of [18], but also to a single-agent double pendulum in simulation. Furthermore, a human-robot virtual reality experiment shows the transferability of the control approach to a human interaction partner, similar to our previous work [18].

In Section II we formally state our problem. The following Section III introduces the essential assumption to extract the intended oscillation by a projection and an approximation step. The progression from the general idea to the intermediate desired oscillation and finally to the control law is described. This is followed by a theoretical discussion of the proposed controller in Section IV. In Section V we apply the control approach to two exemplary pendulum-like objects in simulation and virtual reality and discuss the results. Finally, we draw our conclusions in Section VI.

\section{Problem Formulation}

This paper investigates the problem of controlling the energy contained in oscillations of a general pendulum-like object. We consider pendulum-like objects which can be controlled by several agents and which can have more than only the intended oscillation degree of freedom (DoF). We denote a pendulum with various dominant oscillation DoFs a complex pendulum and we differentiate between single- and multi-agent pendulums.

The intended oscillation DoF is described by the deflection angle $\theta$. The goal is to control the intended oscillation to 
a desired amplitude $\theta_{V}^{\mathrm{d}}$, which represents a desired energy $V_{\theta}^{\mathrm{d}}$. Complex pendulums have additional $n$ disturbance oscillations with deflection angles $\psi_{i}, i \in\{1 \ldots n\}$, which have to be damped. Figure 1 shows two exemplary representatives of complex pendulums, the trapezoidal and the double pendulum. The trapezoidal pendulum is a two-agent pendulum, the double pendulum is a single-agent pendulum, both with one disturbance oscillation $\psi$.

The agents exchange information only mechanically through the pendulum itself. Each agent can manipulate the pendulum through acceleration of his individual handle. In the following, we focus on the view of one agent. For reasons of simplicity and as a minimum requirement we restrict the handle to one-dimensional horizontal movements ( $x$-axis in Fig. 1). The one-dimensional position of the handle is $r$. This results in the decentralized control variable $u=\ddot{r}$.

The agent only gets information about the state of the pendulum through a force sensor which measures the forces applied at the end effector $\boldsymbol{F}$. The agent has to estimate the state of the pendulum from the force $\boldsymbol{F}$ to control the energy of the disturbance oscillations $V_{\psi_{i}}$ to zero and the energy of the desired oscillation $V_{\theta}$ to the desired value $V_{\theta}^{\mathrm{d}}$. Consequently, we are looking for a control law of the following form [18]

$$
\begin{gathered}
u=\ddot{r}(\boldsymbol{F}) \\
\text { with } \quad\left|V_{\theta}^{\mathrm{d}}-V_{\theta}\left(t-t_{0}>T_{s}\right)\right| \leq \epsilon_{\theta} \\
\text { and } \quad\left|0-V_{\psi}\left(t-t_{0}>T_{s}\right)\right| \leq \epsilon_{\psi} \quad \text { for } 0<T_{s}<\infty
\end{gathered}
$$

with the settling time $T_{s}$ and $t_{0}$ being the time at which the controller is engaged. We define a small energy band around the desired energy which has a width of $2 \epsilon_{\theta / \psi}$, where we aim the energy to stay within.

\section{FORCE-TRAJECTORY TRACKING ENERGY CONTROL}

The first Subsection III-A describes how to model the $\theta$ oscillation of the complex pendulum as a simple pendulum through a projection and an approximation step. The general idea, to control the different oscillation DoFs by controlling the measured force to a desired force trajectory, is described in Subsection III-B. Subsection III-C describes how to obtain the desired force trajectory. The last Subsection III-D depicts the entire control law.

\section{A. Approximation as and projection onto simple pendulum}

In this subsection we describe how to extract the desired $\theta$-oscillation of single-/multi-agent complex pendulums through the use of an abstract simple pendulum. We apply two modeling steps: approximation and projection. We proceed in describing the fundamental equations of such an abstract simple pendulum.

We define a world-fixed coordinate system with the $z$-axis pointing in the direction of the initial axis of the desired $\theta$ oscillation. The $y$-axis points upwards and the $x$-axis leads to a right hand coordinate system (see Fig. 1). The first step of gaining the abstract simple pendulum oscillation describing the $\theta$-oscillation is the projection of the multi-pendulum to

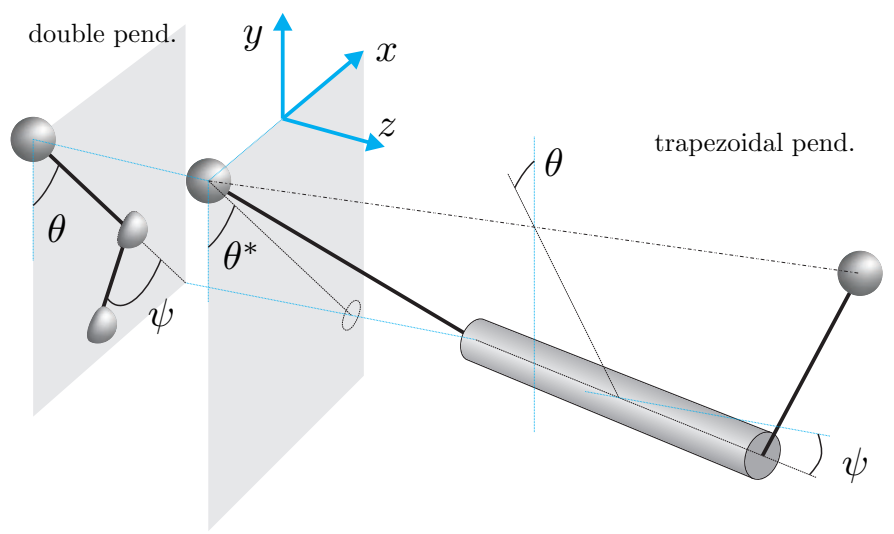

Fig. 1. Setup of double pendulum on the left side, and trapezoidal pendulum on the right side: The deflection angle $\theta$, the disturbance deflection angle $\psi$ and the orthogonal projection resulting in $\theta^{*}$; blue dotted lines are parallel to coordinate axis.

a plane orthogonal to the oscillation axis of the $\theta$-oscillation through the handle. We project the scenery to the $x y$-plane and we obtain the projected deflection angle $\theta^{*}$ from the measured force $\boldsymbol{F}$ as

$$
\theta^{*}=\arctan \frac{-F_{\mathrm{p} x}}{F_{\mathrm{p} y}}
$$

with $\boldsymbol{F}_{\mathrm{p}}=\boldsymbol{F}-m_{\mathrm{h}} \ddot{\boldsymbol{r}}$ being the force compensated for the acceleration of the handle mass $m_{\mathrm{h}}$ and $\ddot{\boldsymbol{r}}=\left(\begin{array}{lll}\ddot{r} & 0 & 0\end{array}\right)^{T}$. The projected oscillation $\theta^{*}$ includes the complete $\theta$-oscillation because of the orthogonal projection, but is superimposed by potentially highly nonlinear projections of the undesired $\psi_{i}$-oscillations.

The second step of gaining the abstract simple pendulum oscillation is the approximation of the desired $\theta$-oscillation as the oscillation of an abstract simple pendulum with deflection angle $\vartheta$. The abstract simple pendulum leads to the possibility to approximately describe the dynamics of the $\theta$-oscillation while being independent of the structure of the complex pendulum and considering only one agent. We virtually mount an abstract simple pendulum with length $l^{*}$ and mass $m_{\mathrm{p}}$ to the handle mass $m_{\mathrm{h}}$. We assume that the influence of the handle motion $\ddot{r}$ on the abstract simple pendulum is limited by the reciprocal number of agents $n_{\mathrm{a}}$. The equation of motion of the abstract simple pendulum results in

$$
\ddot{\vartheta}=-\frac{g}{l^{*}} \sin (\vartheta)-\frac{1}{l^{*}} \cos (\vartheta) \frac{\ddot{r}}{n_{\mathrm{a}}} .
$$

The potential and kinetic energy of the abstract simple pendulum, disregarding the handle motion $\dot{r}$, result in the entire energy of the simple pendulum

$$
V_{\vartheta}=\frac{1}{2} m_{\mathrm{p}} l^{* 2} \dot{\vartheta}^{2}+m_{\mathrm{p}} g l^{*}(1-\cos (\vartheta))
$$

which relates to the amplitude of the oscillation $\vartheta_{V}$ by

$$
V_{\vartheta}=m_{\mathrm{p}} g l^{*}\left(1-\cos \left(\vartheta_{V}\right)\right) \text {. }
$$

The time-derivative of (6) with (5) leads to the energy flow

$$
\dot{V}_{\vartheta}=-m_{\mathrm{p}} l^{*} \cos \vartheta \dot{\vartheta} \frac{\ddot{r}}{n_{\mathrm{a}}},
$$


which can be expressed as the change in amplitude $\left(\vartheta_{V}\right)^{\text {. }}$ through the time-derivative of (7) set equal to (8)

$$
\left(\vartheta_{V}\right)^{\cdot}=-\frac{\dot{\vartheta} \cos \vartheta \ddot{r}}{n_{\mathrm{a}} g \sin \vartheta_{V}} .
$$

Through the abstract simple pendulum we can interpret (8) and (9) as the energy input of our agent.

Centripetal and gravitational forces add up to the simple pendulum force

$$
F_{\vartheta x}=\left(m_{\mathrm{p}} l^{*} \dot{\vartheta}^{2}+m_{\mathrm{p}} g \cos (\vartheta)\right) \sin (\vartheta)
$$

which acts between the rope and the handle for $\dot{r}=0$.

The frequency of the simple pendulum $\omega_{\vartheta}$ is a function of the amplitude $\vartheta_{V}$. Only approximate solutions exist for this function [1]. The small-angle approximation leads to

$$
\omega_{0, \vartheta}=\sqrt{\frac{g}{l^{*}}} .
$$

The exact value of the frequency can be computed by the arithmetic-geometric mean $M\left\{1, \cos \frac{\vartheta_{V}}{2}\right\}$. A sufficiently good approximation is the arithmetic mean [1], which results in

$$
\omega_{\vartheta}=\omega_{0, \vartheta} M\left\{1, \cos \frac{\vartheta_{V}}{2}\right\} \approx \omega_{0, \vartheta} \frac{1+\cos \frac{\vartheta_{V}}{2}}{2} .
$$

For a harmonic oscillation, the trace of $\vartheta$ and $\dot{\vartheta}$ in the state space is elliptic. Norming the angular velocity $\dot{\vartheta}$ with the frequency of the oscillation $\omega_{\vartheta}$ reshapes the ellipse to a circle with constant circulation velocity. This constant circulation velocity $\omega_{\vartheta}$ results in the phase angle $\varphi$ [10]

$$
\varphi:=\arctan 2\left(-\frac{\dot{\vartheta}}{\omega_{\vartheta}}, \vartheta\right),
$$

as illustrated in Fig. 2. The oscillation of the simple pendulum is not harmonic for amplitudes $\vartheta_{V}>0$, but the phase angle $\varphi$ from (13) is still a good approximation for the phase of the oscillation [10] resulting in

$$
\varphi(t)=\int_{0}^{t} \dot{\varphi}(\tau) \mathrm{d} \tau+\varphi_{0} \approx \omega_{\vartheta} t+\varphi_{0}
$$

with $\dot{\varphi} \approx \omega_{\vartheta}$ and the initial phase $\varphi(t=0)=\varphi_{0}$.

\section{B. General idea of the force trajectory tracking controller}

The general idea of the control approach presented in this paper is to control the energies $V_{\theta, \psi_{i}}$ of each oscillations $\theta$ and $\psi_{i}$ through one simple control law, independent of the structure of the pendulum. We assume a unique mapping $f$ from the energy content of each oscillation $\operatorname{DoF} V_{\theta, \psi_{i}}$ and the phase of each DoF $\varphi_{\theta, \psi_{i}}$ to the interaction force trajectory

$$
f: V_{\theta, \psi_{i}} \stackrel{\varphi_{\theta, \psi_{i}}(t)}{\longmapsto} F_{\mathrm{p}, x}(t)=f\left(V_{\theta, \psi_{i}}, \varphi_{\theta, \psi_{i}}(t)\right) .
$$

From this unique mapping we deduce to control the energy of each oscillation DoF $V_{\theta, \psi_{i}}$ by controlling the force trajectory $F_{\mathrm{p}, x}(t)$. By reaching the desired force trajectory $F_{\mathrm{p}, x}^{\mathrm{d}_{\mathrm{i}}}(t)$ the pendulum is reaching the desired energy $V_{\theta, \psi_{i}}^{\mathrm{d}_{\mathrm{i}}}$ of each DoF. This means that controlling the energy by controlling

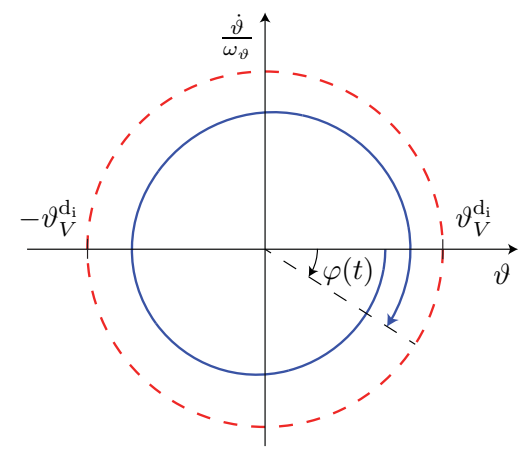

Fig. 2. Phase portrait of a simple pendulum during swing-up resulting in the phase angle $\varphi$. The dashed circle illustrates the intermediate desired oscillation amplitude $\vartheta_{V}^{\mathrm{d}_{\mathrm{i}}}$, to which the actual amplitude $\vartheta_{V}$ converges.

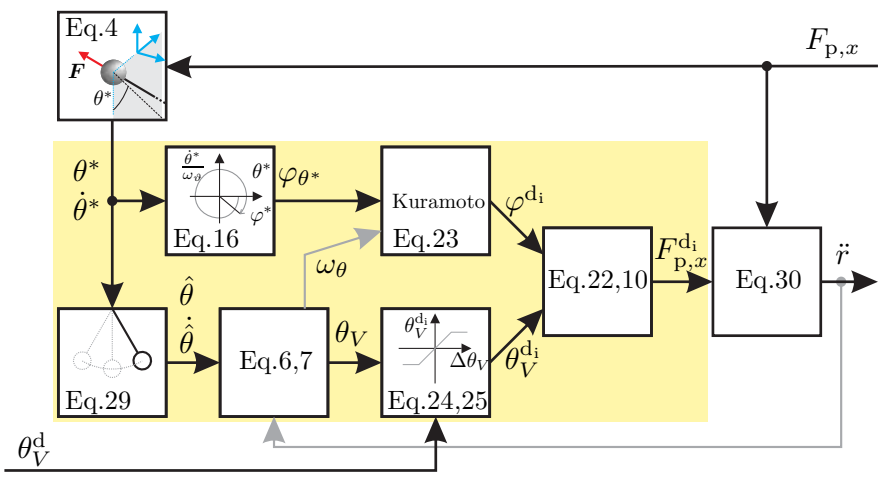

Fig. 3. Control structure for exciting the $\theta$-oscillation: the highlighted area refers to the 'approximation as simple pendulum' gaining the intermediate desired oscillation $\theta^{\mathrm{d}_{\mathrm{i}}}$. The block 'Eq.4' refers to the 'projection onto simple pendulum'. The structure for follower agents $\mathcal{F}$ is not illustrated - the blocks 'Eq.6,7' and 'Eq.24,25' change for the follower.

the force trajectory leads to an automatic allocation of the entire energy flow $\dot{V}$ to the different DoFs $\theta$ and $\psi_{i}$.

We choose the following control law

$$
\dot{r}=k\left(F_{\mathrm{p}, x}^{\mathrm{d}_{\mathrm{i}}}-F_{\mathrm{p}, x}\right),
$$

with the proportional gain $k$ as a design parameter.

Controlling the force by the handle velocity $\dot{r}$ leads to the energy flow $\dot{V}_{\text {app }}$ applied by the agent to the pendulum

$$
\dot{V}_{\text {app }}=\sum_{j=\theta, \psi_{i}} \dot{V}_{j}=\dot{r} F_{\mathrm{p}, x} .
$$

The proportional force control law (16) combined with the unique mapping (15) and the applied energy flow (17) leads to the energy dynamics

$\dot{V}_{\text {app }}=k\left[f\left(V_{\theta, \psi_{i}}^{\mathrm{d}_{\mathrm{i}}}, \varphi_{\theta, \psi_{i}}\right)-f\left(V_{\theta, \psi_{i}}, \varphi_{\theta, \psi_{i}}\right)\right] f\left(V_{\theta, \psi_{i}}, \varphi_{\theta, \psi_{i}}\right)$,

which consists of the difference between two quadratic force terms $f\left(V_{\theta, \psi_{i}}^{\mathrm{d}_{\mathrm{i}}}, \varphi_{\theta, \psi_{i}}\right) f\left(V_{\theta, \psi_{i}}, \varphi_{\theta, \psi_{i}}\right)$ and $f\left(V_{\theta, \psi_{i}}, \varphi_{\theta, \psi_{i}}\right)^{2}$. In the following subsection we describe how to obtain the intermediate desired oscillation force trajectory $F_{\mathrm{p}, x}^{\mathrm{d}_{\mathrm{i}}}$.

\section{Online computed intermediate desired oscillation}

As described in the last subsection we control the system energy by controlling the force to an intermediate desired 
force $F_{\mathrm{p}, x}^{\mathrm{d}_{\mathrm{i}}}$. The term 'intermediate' is indicated by the superscript $d_{i}$ and refers to a desired oscillation which pulls the actual oscillation to the final desired oscillation in (2), to be the final goal, indicated by the superscript $d$. The desired force $F_{\mathrm{p}, x}^{\mathrm{d}_{\mathrm{i}}}$ is obtained through (10) with a desired oscillation deflection angle $\theta^{\mathrm{d}_{\mathrm{i}}}$. As discussed in Sec. III-A the desired oscillation is approximated by a simple pendulum oscillation with equal parameters as in (5). The desired oscillation can be described by its phase $\varphi^{\mathrm{d}_{\mathrm{i}}}$ and its amplitude $\theta_{V}^{\mathrm{d}_{\mathrm{i}}}$. Figure 3 illustrates how to obtain the phase of the desired oscillation $\varphi^{\mathrm{d}_{\mathrm{i}}}$ in the upper path and the amplitude $\theta_{V}^{\mathrm{d}_{\mathrm{i}}}$ in the lower path in the highlighted area. We obtain the deflection angle and its derivative of the desired oscillation through

$$
\theta^{\mathrm{d}_{\mathrm{i}}}=\cos \varphi^{\mathrm{d}_{\mathrm{i}}} \theta_{V}^{\mathrm{d}_{\mathrm{i}}} \quad, \quad \dot{\theta}^{\mathrm{d}_{\mathrm{i}}}=-\sin \varphi^{\mathrm{d}_{\mathrm{i}}} \theta_{V}^{\mathrm{d}_{\mathrm{i}}} \omega_{\theta}
$$

with the approximation $\dot{\varphi}^{\mathrm{d}_{\mathrm{i}}}=\omega_{\theta}=$ const and $\theta_{V}^{\mathrm{d}_{\mathrm{i}}}=\mathrm{const}$ (see Fig. 2). The desired oscillation synchronizes to the $\theta$-oscillation through $\varphi^{\mathrm{d}_{\mathrm{i}}} \approx \varphi_{\theta}$. The desired amplitude $\theta_{V}^{\mathrm{d}_{\mathrm{i}}}$ pulls the amplitude of the $\theta$-oscillation to the desired amplitude $\theta_{V}^{\mathrm{d}}$, which is the final goal.

We extract the phase of the $\theta$-oscillation $\varphi_{\theta}$ and assign it to the phase of the desired oscillation $\varphi^{\mathrm{d}_{\mathrm{i}}}$. The projected oscillation $\theta^{*}$ is the $\theta$-oscillation superimposed with nonlinear projections of disturbance oscillations $\psi_{i}$. As we assume the $\theta$-oscillation to be similar to a simplependulum oscillation we compute the projected phase $\varphi^{*}$ through (13) from the projected deflection angle $\theta^{*}$ and the frequency $\omega_{\theta}$. This frequency $\omega_{\theta}$ is obtained through (12) with the amplitude $\theta_{V}$. The phase $\varphi^{*}$ consists of the phase of the $\theta$-oscillation but superimposed with highly nonlinear phases of the disturbance oscillations $\psi_{i}$. A nonlinear filter based on the Kuramoto-equation [19]

$$
\dot{\varphi}^{\mathrm{d}_{\mathrm{i}}}=\omega_{\theta}+\kappa \sin \left(\varphi^{*}-\varphi^{\mathrm{d}_{\mathrm{i}}}\right)
$$

leads to the desired phase which is approximately equal to the phase of the $\theta$-oscillation $\varphi^{\mathrm{d}_{\mathrm{i}}} \approx \varphi_{\theta}$ [18].

We obtain the amplitude of the desired oscillation $\theta_{V}^{\mathrm{d}_{\mathrm{i}}}$ from the amplitude of the $\theta$-oscillation $\theta_{V}$ modified by an additive term $\Delta \bar{\theta}_{V}$ resulting in

$$
\theta_{V}^{\mathrm{d}_{\mathrm{i}}}=\theta_{V}+\Delta \bar{\theta}_{V}
$$

We differentiate between leader agents $\mathcal{L}$ who know the final desired amplitude $\theta_{V}^{\mathrm{d}}$ and follower agents $\mathcal{F}$ who imitate the behavior of the other agents.

Leader agents $\mathcal{L}$ know the final desired amplitude $\theta_{V}^{\mathrm{d}}$, hence we choose $\Delta \bar{\theta}_{V}$ of (21) in a way that the amplitude of the intermediate desired oscillation $\theta_{V}^{\mathrm{d}_{\mathrm{i}}}$ approaches the final desired amplitude $\theta_{V}^{\mathrm{d}}$. We restrict the control variable $u$ by saturating the amplitude control error $\Delta \theta_{V}=\theta_{V}^{\mathrm{d}}-\theta_{V}$ to the parameter $\Delta_{\mathcal{L}}$ as

$$
\mathcal{L}: \Delta \bar{\theta}_{V}= \begin{cases}\Delta_{\mathcal{L}} \operatorname{sgn}\left(\Delta \theta_{V}\right) & \text { if }\left|\Delta \theta_{V}\right| \geq \Delta_{\mathcal{L}} \\ \Delta \theta_{V} & \text { else }\end{cases}
$$

Follower agents $\mathcal{F}$ do not know the final desired amplitude $\theta_{V}^{\mathrm{d}}$, but they support the external energy flow, caused by the other agents. The time-derivative of (7) $\dot{V}_{\vartheta}=$ $m_{\mathrm{p}} g l^{*} \sin \vartheta_{V}\left(\vartheta_{V}\right)^{\cdot}$ shows a linear relation between energy flow $\dot{V}_{\vartheta}$ and change in amplitude $\left(\vartheta_{V}\right)^{\text {: }}$. From this linearity we deduce additivity of the entire change in amplitude

$$
\left(\theta_{V}\right)^{\cdot}=\left(\theta_{V}\right)_{\mathcal{F}}^{\cdot}+\left(\theta_{V}\right)_{\text {ext }},
$$

with the follower's change in amplitude $\left(\theta_{V}\right)_{\mathcal{F}}$ and the external change in amplitude $\left(\theta_{V}\right)_{\text {ext }}^{*}$ caused predominantly by the other agents. From this we introduce a saturated proportional law such that the additive term of the desired oscillation amplitude in (21) is

$$
\mathcal{F}: \Delta \bar{\theta}_{V}= \begin{cases}\Delta_{\mathcal{F}} \operatorname{sgn}\left(\left(\theta_{V}\right)_{\text {ext }}^{\cdot}\right) & \text { if } k_{\Delta}\left|\left(\theta_{V}\right)_{\text {ext }}^{\cdot}\right| \geq \Delta_{\mathcal{F}} \\ k_{\Delta}\left(\theta_{V}\right)_{\text {ext }}^{\cdot} & \text { else }\end{cases}
$$

with the proportional gain $k_{\Delta}$ and the saturation at $\Delta_{\mathcal{F}}$.

The external change in amplitude $\left(\theta_{V}\right)_{\text {ext }}^{*}$ is obtained through (23) with the entire change in amplitude $\left(\theta_{V}\right)^{\circ}$ and the follower's change in amplitude $\left(\theta_{V}\right)_{\mathcal{F}}{ }_{\mathcal{F}}$. We obtain the entire change in amplitude $\left(\theta_{V}\right)^{\circ}$ by differentiating the amplitude $\theta_{V}$ with the following filter

$$
\left(\theta_{V}\right)^{\cdot}=\frac{s}{T_{F}^{2} s^{2}+2 T_{F} s+1} \theta_{V}(s)
$$

which differentiates up to the frequency $\frac{1}{T_{F}}$ and damps higher frequencies. Considering the simple-pendulum approximation we use (9) and the filter of (25) with the numerator equal to 1 , to compute the follower's change in amplitude $\left(\theta_{V}\right)_{\mathcal{F}}^{\circ}$ depending on the amplitude of the $\theta$ oscillation $\theta_{V}$.

We compute the amplitude of the $\theta$-oscillation $\theta_{V}$ based on the abstract simple pendulum approximation by setting (6) equal to (7). The deflection angle $\theta$ is obtained through a nonlinear observer [18] with state vector $\hat{\boldsymbol{\theta}}=\left[\begin{array}{ll}\hat{\theta} & \dot{\hat{\theta}}\end{array}\right]^{T} \approx$ $\left[\begin{array}{ll}\theta & \dot{\theta}\end{array}\right]^{T}$ which yields approximately to the $\theta$-oscillation. The dynamical equation of the observer is

$$
\dot{\hat{\boldsymbol{\theta}}}=\left[\begin{array}{c}
\dot{\hat{\theta}} \\
-\frac{g}{l^{*}} \sin (\hat{\theta})
\end{array}\right]+\boldsymbol{L}\left(\theta^{*}-y\right) \quad y=\left[\begin{array}{ll}
1 & 0
\end{array}\right] \hat{\boldsymbol{\theta}},
$$

which consists of the abstract simple pendulum dynamics (5) and the term $L\left(\theta^{*}-y\right)$, which couples the observer to the real pendulum. The state of the observer approximates the abstract simple pendulum deflection angle $\vartheta \approx \hat{\theta}$ which allows to compute the frequency $\omega_{\theta}$ through (12) and amplitude $\theta_{V}^{\mathrm{d}_{\mathrm{i}}}$ of the desired oscillation $\theta^{\mathrm{d}_{\mathrm{i}}}$ through (21). These computations use the oscillation amplitude $\theta_{V}$ which gets computed through (6) and (7) with $\vartheta \approx \hat{\theta}$.

\section{Control law}

The control law is based on the proportional force control law (16). Consequently, the handle velocity $\dot{r}$ equals a proportional gain $k$ times the difference between the desired force trajectory $F_{\mathrm{p}, x}^{\mathrm{d}_{\mathrm{i}}}$ and the actual force $F_{\mathrm{p}, x}$. For a position controlled robot, we control it to track the handle position $r$. The handle position $r$ consists of the control law mentioned 
in (16) but integrated to the position level with an additional band-pass filter. The entire control law results in

$$
r(s)=k \frac{1}{1+T_{\mathrm{lp}} s} \frac{T_{\mathrm{hp}} s}{1+T_{\mathrm{hp}} s} \frac{1}{s}\left(F_{\mathrm{p}, x}^{\mathrm{d}_{\mathrm{i}}}-F_{\mathrm{p}, x}\right)
$$

with the band-pass filter consisting of a first order high-pass filter with time constant $T_{\mathrm{hp}}$ and a first order low-pass filter with time constant $T_{\mathrm{lp}}$. The purpose of the low-pass filter is to filter out high frequencies contained in the force signal $F_{\mathrm{p}, x}$ and the high-pass filter to avoid drift due to the time integration from handle velocity $\dot{r}$ to position $r$.

\section{DISCUSSION OF THE CONTROL METHOD}

The swing-up control approach described in Sec. III is based on two essential assumptions: first to model the desired $\theta$-oscillation $\theta^{\mathrm{d}_{\mathrm{i}}}$ as a simple pendulum oscillation and second the unique mapping $f\left(V_{\theta, \psi_{i}}, \varphi_{\theta, \psi_{i}}\right)$ from the energy content and phase of each oscillation DoF to the force trajectory (15).

\section{A. Discussion of the simple pendulum approximation}

In Sec. III-A we model the desired $\theta$-oscillation as a simple pendulum oscillation. This leads to several restrictions for a general pendulum. We require the complex pendulum to be a gravity pendulum, which behaves similar to a simple pendulum with respect to the $\theta$-oscillation. If this constraint is not sufficiently met the abstract simple pendulum approximation becomes invalid. Deviations of the behavior of a complex pendulum $\theta$-oscillation from a simple pendulum oscillation have negative influence on the control performance. This limitation becomes apparent for the double-pendulum as investigated in V-B and V-C.

There are two purposes for the simple pendulum projection and approximation. First, we observe the amplitude of the $\theta$ oscillation by the nonlinear observer based on the differential equation of the simple pendulum (26). Second, we gain the intermediate desired oscillation $\theta^{\mathrm{d}_{\mathrm{i}}}$ based on the simple pendulum approximation. Without this simple pendulum approximation we cannot observe the actual $\theta$-oscillation and cannot compute online the desired oscillation $\theta^{\mathrm{d}_{\mathrm{i}}}$.

\section{B. Discussion of the unique mapping}

In Sec. III-B we argue that there is a unique mapping from the energy content and phase of each oscillation DoF to the force trajectory (15). The validity of this unique mapping cannot be proved in general.

The unique mapping maps the energies of each $\operatorname{DoF} V_{\theta, \psi_{i}}$ with the appropriate phases to the force trajectory $F_{\mathrm{p}, x}(t)$ by neglecting the influence of handle motion $\dot{r}$. The energies $V_{\theta, \psi_{i}}$ and phases $\varphi_{\theta, \psi_{i}}$ span a transformed state space. This transformed state space is equivalent to the state space consisting of the deflection angles $\theta$ and $\psi_{i}$ with its timederivatives. The unique mapping is highly related to the concept of observability. Observability means that we are able to obtain the entire state of a dynamical system from the system output in finite time. We are interested in the energies $V_{\theta, \psi_{i}}$ which we obtain from the output of the complex pendulum system, which is the force $F_{\mathrm{p}, x}(t)$. The energies $V_{\theta, \psi_{i}}$ are a strict subset of the entire state.
In contrast to the undirected quantities energies $V_{\theta, \psi_{i}}$, the force $F_{\mathrm{p}, x}(t)$ is a directed quantity as long as it attains positive and negative values. This means that the unique mapping (15) maps the undirected quantities, the energies $V_{\theta, \psi_{i}}$, to the directed quantity, the force $F_{\mathrm{p}, x}(t)$.

The applied energy dynamics (18) are obtained by the unique mapping (15) under negligence of the handle motion $\dot{r}$. This is justified by assuming a bigger influence of the control variable $\dot{r}$ on the force than directly on the mapping $f\left(V_{\theta, \psi_{i}}, \varphi_{\theta, \psi_{i}}\right)$ and by the indirect restrictions of the control variable $\dot{r}$ through (22) and (24). Multiplying the directed force terms $f\left(V_{\theta, \psi_{i}}, \varphi_{\theta, \psi_{i}}\right)$ and $f\left(V_{\theta, \psi_{i}}^{\mathrm{d}_{\mathrm{i}}}, \varphi_{\theta, \psi_{i}}^{\mathrm{d}_{\mathrm{i}}}\right)$ with $f\left(V_{\theta, \psi_{i}}, \varphi_{\theta, \psi_{i}}\right)$ transforms the directed force terms to undirected quadratic quantities like the energy $V_{\theta, \psi_{i}}$. The difference between both quadratic terms indicates to increase, decrease or hold the energy as long as the mapping $f$ is strictly monotonic. It is essential for canceling out the directive component in $f\left(V_{\theta, \psi_{i}}^{\mathrm{d}_{\mathrm{i}}}, \varphi_{\theta, \psi_{i}}^{\mathrm{d}_{\mathrm{i}}}\right) f\left(V_{\theta, \psi_{i}}, \varphi_{\theta, \psi_{i}}\right)$ that the desired phase $\varphi^{\mathrm{d}_{\mathrm{i}}}$ is synchronized to the actual phase $\varphi_{\theta}$. This synchronization means the same directive component of $f\left(V_{\theta, \psi_{i}}^{\mathrm{d}_{\mathrm{i}}}, \varphi_{\theta, \psi_{i}}^{\mathrm{d}_{\mathrm{i}}}\right)$ and $f\left(V_{\theta, \psi_{i}}, \varphi_{\theta, \psi_{i}}\right)$ for low energies of the disturbance oscillation $V_{\psi_{i}}$.

\section{EXPERIMENTAL EVALUATION}

Two examples for complex pendulums are the trapezoidal pendulum as a two-agent pendulum with one predominant disturbance oscillation [18] and the double pendulum as a single agent pendulum with a highly nonlinear disturbance oscillation as illustrated in Fig. 1.

The first two experiments are a robot-robot simulation experiment and a human-robot virtual reality experiment with the trapezoidal pendulum. Simulation based experiments are reproducible and we can compare the results of this experiment to the results of our previous control approach [18]. The human-robot experiment shows the transferability of the control approach to a human interaction partner. The second experiment is a robotic simulation based experiment with a double pendulum to investigate the flexibility and generalizability of this control approach, as well as its limitations.

We use the same control parameters for all experiments. The controller is tuned to obtain results comparable to [18]. The control parameters as well as the parameters of the abstract simple pendulum are listed in Table I.

TABLE I

CONTROL PARAMETERS

\begin{tabular}{cccccc}
$\Delta_{\mathcal{L}}[\mathrm{rad}]$ & $\Delta_{\mathcal{F}}[\mathrm{rad}]$ & $k_{\Delta}[\mathrm{s}]$ & $k_{\mathcal{L}}[\mathrm{s} / \mathrm{kg}]$ & $k_{\mathcal{F}}[\mathrm{f}]$ & $m_{\mathrm{p}}[\mathrm{kg}]$ \\
\hline 0.1388 & 0.16 & 1.9 & 0.15 & 0.18 & 2.5 \\
& & & & & \\
$L_{1}[1 / \mathrm{s}]$ & $T_{\mathrm{F}}$ & $\kappa[\mathrm{rad} / \mathrm{s}]$ & $T_{\mathrm{lp}}[\mathrm{s}]$ & $T_{\mathrm{hp}}[\mathrm{s}]$ & $\omega_{0, \theta}[\mathrm{rad} / \mathrm{s}]$ \\
\hline$\omega_{\theta}$ & $\frac{1.5}{\omega_{0, \theta}}$ & $\omega_{0, \theta}$ & $\frac{1}{\omega_{0, \theta}}$ & $\frac{2}{\omega_{0, \theta}}$ & 4
\end{tabular}

\section{A. Trapezoidal pendulum experiments}

The two-agent trapezoidal pendulum [18] illustrated in Fig. 1 consists of a cylindrical pendulum object with 
mass $2 m_{\mathrm{p}}$. We assume one predominant disturbance oscillation $n=1$ with deflection angle $\psi$ as the angle between the cylindrical object and the $z$-axis. The angle between the $y$ axis and the connecting line of the center of mass of the two handles and the center of mass of the cylindrical object is the deflection angle $\theta$ of the desired oscillation. The pendulum is controlled by two agents $n_{\mathrm{a}}=2$ and the projection to the $x y$-plane leads to a simple pendulum for $\psi=0$. Further descriptions and parameters are given in [18].

Referring to the simulation based experiment, we analyze the performance of the control approach for two different initial deflection angles: $\theta_{0,1}=10^{\circ}, \psi_{0,1}=0^{\circ}$ and $\theta_{0,2}=5.7^{\circ}, \psi_{0,2}=19.4^{\circ}$. The final goal is $\theta_{V, 1}^{\mathrm{d}}=$ $\theta_{V, 2}^{\mathrm{d}}=45^{\circ}$. For the performance analysis we compute the settling time $T_{\mathrm{s}}$ until the system energy $V$ stays within $2 \%$ of its steady-state value. Furthermore we compute the steady-state error $e_{\mathrm{ss}}$. The effort sharing is indicated by the relative follower contribution $R C_{\mathcal{F}}$ as the relation between the amount of energy applied by the follower and the applied energy of the leader and follower $R C_{\mathcal{F}}=V_{\mathcal{F}}\left(t_{0}+\right.$ $\left.T_{\mathrm{s}}\right)\left(V_{\mathcal{F}}\left(t_{0}+T_{\mathrm{s}}\right)+V_{\mathcal{L}}\left(t_{0}+T_{\mathrm{s}}\right)\right)^{-1}$ with $t_{0}=3 \mathrm{~s}$ as the time at which the two controllers are engaged.

The steady-state errors are $e_{\mathrm{ss}, 1}=e_{\mathrm{ss}, 2}=0.7 \%$. The settling times are $T_{\mathrm{s}, 1}=12.45 \mathrm{~s}$ and $T_{\mathrm{s}, 2}=14.76 \mathrm{~s}$. The relative follower contributions are $R C_{\mathcal{F}, 1}=53.88 \%$ and $R C_{\mathcal{F}, 2}=58.11 \%$. The amplitude of the remaining disturbance oscillations are $\psi_{V, \infty, 1}=\psi_{V, \infty, 2}=0.053^{\circ}$. For brevity, we only illustrate the simulation results for $\theta_{0,2}=$ $5.7^{\circ}, \psi_{0,2}=19.4^{\circ}$ in Fig. 4 . The first graph illustrates the energies over time. The entire energy $V$ and the energy $V_{\theta}$ computed through (6) with the real deflection angle $\theta$ show fast settling with low overshoot. Furthermore, as expected, the follower reacts slower than the leader. The second graph illustrates the deflection angles $\theta$ and $\psi$. We observe fast damping of the disturbance oscillation $\psi$ followed by a significant increase of the energy of the $\theta$-oscillation $V_{\theta}$. The third graph illustrates the energy flow to the complex pendulum of the leader $\dot{V}_{\text {app, } \mathcal{L}}$ and the follower's estimation of it as the external energy input $\dot{V}_{\theta \text {,ext }}$. This external energy input $\dot{V}_{\theta \text {,ext }}$ is obtained through (7) and its timederivative as $\dot{V}_{\theta \text {,ext }}=m_{\mathrm{p}} g l^{*} \sin \left(\arccos \left(1-\frac{V_{\theta}}{m_{\mathrm{p}} g l^{*}}\right)\right)\left(\theta_{V}\right)_{\text {ext }}^{\dot{ }}$. The last graph illustrates the handle movements with maximum amplitude $r_{\max }=0.15 \mathrm{~m}$.

We apply the same trapezoidal-pendulum experiment to a human-robot virtual reality experiment to show the transferability of this control approach to the cooperation of a human and a robot. The experimental setup is based on a virtual reality environment. The human interacts with the virtual reality through a haptic device. The setup is explained in detail in [17]. The trapezoidal pendulum is identical to that of the simulation based experiment of this section. The initial deflection angles are $\theta_{0,3}=0^{\circ}$ and $\psi_{0,3}=0^{\circ}$. The final goal is $\theta_{V, 3}^{\mathrm{d}}=45^{\circ}$. The human acts as a leader and the robot as a follower agent. The desired deflection angle $\theta_{V, 3}^{\mathrm{d}}$ as well as the entire scenery are shown on a screen to give visual feedback of the swinging process to the human.
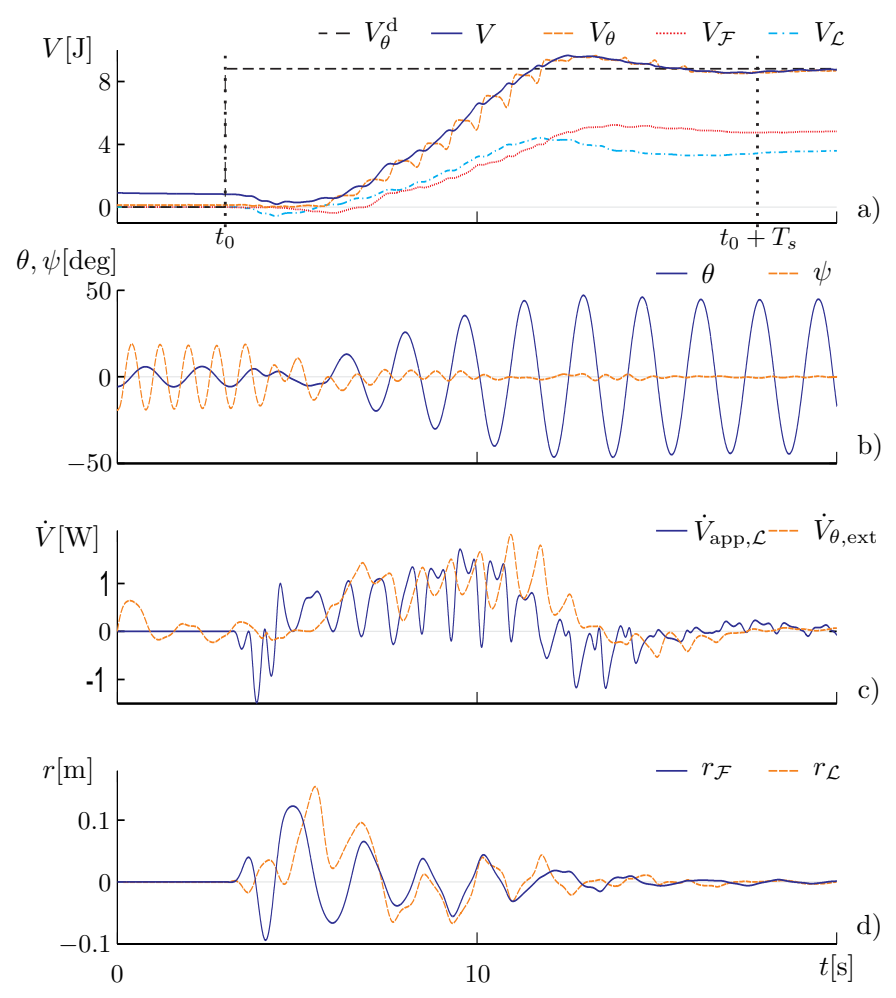

Fig. 4. Results of swing-up experiment of trapezoidal pendulum with initial conditions $\theta_{0,2}=5.7^{\circ}, \psi_{0,2}=19.4^{\circ}$ and goal $\theta_{V}^{\mathrm{d}}=45^{\circ}$ : a) energies $V$, b) deflection angles $\theta$ and $\psi$, c) energy flow of leader $\dot{V}_{\text {app, } \mathcal{L}}$ and its estimation $\dot{V}_{\theta, \text { ext }}$, d) handle trajectory $r$.

The results of the human-robot virtual reality experiment are illustrated in form of energies over time in Fig. 5. We observe a similar settling time as in the simulation based experiment, without overshoot. The contribution of the follower is approximately $50 \%$. The accuracy of the human-robot experiment is low, in comparison to the robotrobot experiment. The second graph illustrates the deflection angles $\theta$ and $\psi$. The disturbance oscillation $\psi$ stays within the limits of $\pm 5^{\circ}$. The intended oscillation $\theta$ reaches the desired amplitude $\theta_{V, 3}^{\mathrm{d}}$.

\section{B. Double pendulum experiment}

The double pendulum experiment shows the limitations of the control approach with respect to its generalizability. The double pendulum illustrated in Fig. 1 is a simple pendulum with mass $m_{2}$ and length $l_{2}$ mounted at the end of another simple pendulum with mass $m_{1}$ and length $l_{1}$. The upper pendulum is referred to by the index 1 . The angle between both pendulums is the disturbance angle $\psi$. We define the desired oscillation of the double pendulum to be the oscillation with stretched lever, meaning $\psi=0$. The dynamic equation of the double pendulum with fixed lever $(\psi=0 \forall t)$ is

$$
\ddot{\theta}=-\frac{m_{1}+m_{2}}{l_{1} m_{1}+\left(l_{1}+l_{2}\right) m_{2}}(g \sin \theta+\ddot{r} \cos \theta) .
$$



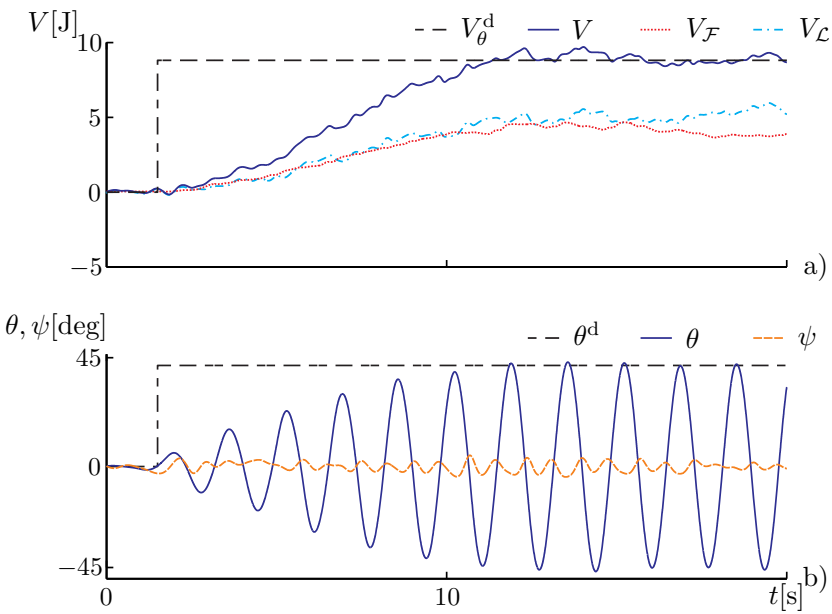

Fig. 5. Results of the human-robot virtual reality experiment with initial conditions $\theta_{0,3} \approx 0^{\circ}, \psi_{0,3} \approx 0^{\circ}$ and goal $\theta_{V}^{\mathrm{d}}=45^{\circ}$ : a) energies $V$ and b) deflection angles $\theta$ and $\psi$. The leader agent is the human.

We obtain the same differential equation for the dynamics of the double pendulum with fixed stretched lever as the differential equation of the simple pendulum (5) with

$$
\frac{m_{1}+m_{2}}{l_{1} m_{1}+\left(l_{1}+l_{2}\right) m_{2}} \stackrel{!}{=} \frac{1}{l^{*}},
$$

$n_{\mathrm{a}}=1$ and the masses of the double pendulum $m_{1}+m_{2}=$ $m_{\mathrm{p}}$. The other system parameters and the control parameters used in the simulation of the double pendulum are equal to those of the trapezoidal pendulum.

The simulation of the double pendulum is conducted for masses $m_{1}=m_{2}=1.25 \mathrm{~kg}$ and lengths of the levers $l_{1} \approx 0.526 \mathrm{~m}$ and $l_{2} \approx 0.175 \mathrm{~m}$, resulting in $\frac{l_{1}}{l_{2}}=\frac{3}{1}$. The initial deflection angles are $\theta_{0,3}=10^{\circ}$ and $\psi_{0,3}=110^{\circ}$. Consequently, the simulation starts with an excessive deflection of the disturbance oscillation. The simulation leads to the resulting deflection angles illustrated in Fig. 6. After settling, we obtain an alternating amplitude $\theta_{V}$, which stays below $\Delta \theta_{V, \infty, 3} \leq 10^{\circ}$. The amplitude of the remaining disturbance oscillation is $\psi_{V, \infty, 3} \approx 50^{\circ}$. Note that both oscillation DoFs influence each other drastically (see uncontrolled first seconds $t<t_{0}=3 \mathrm{~s}$ ).

\section{Discussion of experiments}

First, we analyze the performance of reaching the task goal stated in (2) and (3). For the trapezoidal pendulum we reach both control goals, swinging up $\theta$ to the final desired amplitude $\theta_{V}^{\mathrm{d}}$ and damping $\psi$, with low steady state errors. The predominant causes for the steady-state errors of the robot-robot experiments are first the damping of the system, which is not considered in the control approach, second the only approximately orthogonal projection combined with the simple pendulum approximation and third the unwanted excitation of the disturbance oscillation $\psi$ while compensating for energy losses due to damping. The temporal lag of the follower agent versus the leader causes an overshoot, but has no influence on the steady-state, because
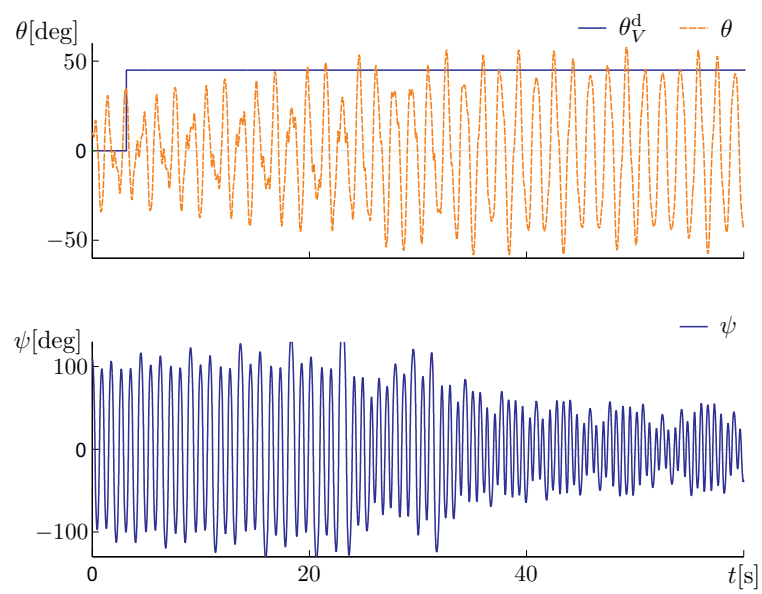

Fig. 6. Results of swing-up experiment of double pendulum with initial conditions $\theta_{0,3}=10^{\circ}, \psi_{0,3}=110^{\circ}$ and goal $\theta_{V}^{\mathrm{d}}=45^{\circ}$ : the deflection angles $\theta$ to be excited and $\psi$ to be damped.

both agents synchronize with the mechanical pendulum and this synchronization is not affected by this lag.

The leader achieves a rapid decrease of the initial disturbance oscillation $\psi$ within the first $2 \mathrm{~s}$ (see Fig. $4 \mathrm{~b}$ ) and c)). Because the time constants are too long and the follower only observes the $\theta$-oscillation, the follower does not detect the leader's energy extraction between $t=3 \mathrm{~s}$ and $t=5 \mathrm{~s}$. The applied energy flow of each agent is not easily relatable to the oscillation DoFs $\theta$ and $\psi$.

The settling time $T_{\mathrm{s}, 2}=14.76 \mathrm{~s}$ of the experiment with the initial disturbance oscillation $\psi_{0,2}=19.4^{\circ}$ is about $2 \mathrm{~s}$ longer than the settling time $T_{\mathrm{s}, 1}=12.45 \mathrm{~s}$ of the experiment without initial disturbance oscillation. Various simulations showed that this is predominantly caused by the initial disturbance oscillation of $\psi_{0,2}-\psi_{0,1}=19.4^{\circ}$ and not by the lower initial $\theta$-oscillation of $\theta_{0,2}-\theta_{0,1}=4.3^{\circ}$. The controller first extracts the energy contained in the $\psi$-oscillation and afterwards injects the energy to the $\theta$-oscillation which stimulates the $\psi$-oscillation, such that this has to be damped simultaneously.

The simulation experiment with the tapezoidal pendulum shows comparable results to the experiment of our previous work [18]. An essential difference to our previous work [18] is that this controller is not based on model parameters except of the parameters of the abstract simple pendulum. Our previous work is additionally based on a model of the disturbance oscillation $\psi$.

The results of the human-robot experiment are highly dependent on the performance of the human, because the robotic follower only reinforces the behavior of the human leader. The lower accuracy of the human-robot experiment is caused by the lower accuracy of the human as the leading agent.

The double-pendulum experiment illustrated in Fig. 6 shows poorer results than the trapezoidal pendulum experiment. The amplitude of the remaining disturbance oscillation of the double pendulum experiment is $\psi_{V, \infty, 3} \approx$ $50^{\circ}$ compared to the trapezoidal pendulum with $\psi_{V, \infty, 1}=$ $\psi_{V, \infty, 2}=0.053^{\circ}$. The predominant reason for this is that 
both oscillations $\theta$ and $\psi$ interchange energy rapidly for the double pendulum, but not for the trapezoidal pendulum. In all experiments the controller is activated after $t_{0}=3 \mathrm{~s}$. For the trapezoidal pendulum in Fig. 4 b) we perceive a low influence of both oscillations onto each other, in contrast to the double pendulum in Fig. 6. The two DoFs $\theta$ and $\psi$ of the double pendulum interchange energy instantaneously, causing simultaneous damping of $\psi$ and excitation of $\theta$. This leads to a longer duration for the swing-up of the double pendulum, compared to the trapezoidal pendulum.

A further reason for the poorer results of the doublependulum is that the force trajectory of a double pendulum does not match the one of a simple pendulum, even with $\psi=0^{\circ} \forall t$ due to the distributed mass. The choice that the relation between both levers of the double-pendulum is $\frac{l_{1}}{l_{2}}=$ $\frac{3}{1}$ justifies the simple-pendulum approximation. This leads to further restrictions for the generalizability of this control approach. The general complex pendulum is restricted to pendulums whose desired oscillation DoF behaves similar to a simple pendulum with limited influence of disturbance oscillations on the desired oscillation.

\section{CONCLUSION}

This paper investigates the energy control of complex pendulums by tracking an online computed force trajectory. The control approach is based on an unique mapping of the energy content of different oscillation DoFs to the force trajectory. We extract the intended oscillation DoF by a simple pendulum approximation.

The presented approach allows to excite the intended oscillation DoF to the desired amplitude, while damping all other oscillation DoFs. However, the intended DoF is restricted to be similar to a simple pendulum oscillation, meaning that the pendulum-like object can swing around a horizontal axis, like a simple pendulum. The convincing results of the robotrobot and the human-robot trapezoidal pendulum experiments show the practicability of the underlying assumptions of our approach, like the unique mapping. The simulation experiment with the double pendulum with strongly coupled oscillation DoFs shows the limits of generalizability of the proposed control approach.

This control approach is applicable to different tasks. Exemplary tasks are described in the introduction, like damping of residual oscillations during transport of slung-loads or swing-up tasks like lifting a sports mat onto a trolley. The human-robot experiment shows the transferability of this control approach to human-robot cooperative swinging of pendulum-like objects. In contrast to other swinging control approaches, this force-trajectory tracking control approach directly controls the interaction forces, which aims for using a position controlled robot with force feedback.

In future work we plan to extend the control approach towards adapting to the pendulum parameters. Finally, we want to use the results of pendulum-like object swinging to enable cooperative swinging of bulky, flexible objects.

\section{REFERENCES}

[1] C. Carvalhaes and P. Suppes, "Approximations for the period of the simple pendulum based on the arithmetic-geometric mean," Am. J. Phys., vol. 76, no. 12, pp. 1150-1154, 2008.

[2] S. Kajita and K. Tani, "Study of dynamic biped locomotion on rugged terrain-theory and basic experiment," in Proc. IEEE ICAR, vol. 1, 1991, pp. 741-746.

[3] S. Kajita, F. Kanehiro, K. Kaneko, K. Yokoi, and H. Hirukawa, "The 3d linear inverted pendulum mode: a simple modeling for a biped walking pattern generation," in Proc. IEEE IROS, vol. 1, 2001, pp. 239-246 vol.1.

[4] H. Wang and K. Kosuge, "Control of a robot dancer for enhancing haptic human-robot interaction in waltz," IEEE Trans. on Haptics, vol. 5, no. 3, pp. 264-273, Quarter.

[5] K. Yano and K. Terashima, "Robust liquid container transfer control for complete sloshing suppression," IEEE Trans. on Control Systems Technology, vol. 9, no. 3, pp. 483-493, 2001.

[6] J. Nakanishi, T. Fukuda, and D. Koditschek, "A brachiating robot controller," IEEE Trans. on Robotics and Automation, vol. 16, no. 2, pp. 109-123, 2000.

[7] D. Richiedei and A. Trevisani, "Delayed-reference anti-swing control of overhead crane systems," in IEEE Int. Workshop on Advanced Motion Control, 2008, pp. 92-97.

[8] I. Palunko, R. Fierro, and P. Cruz, "Trajectory generation for swingfree maneuvers of a quadrotor with suspended payload: A dynamic programming approach," in Proc. IEEE ICRA, 2012, pp. 2691-2697.

[9] D. Zameroski, G. Starr, J. Wood, and R. Lumia, "Swing-free trajectory generation for dual cooperative manipulators using dynamic programming," in Proc. IEEE ICRA, 2006, pp. 1997-2003.

[10] K. Yoshida, "Swing-up control of an inverted pendulum by energybased methods," in Proc. ACC, vol. 6, 1999, pp. 4045-4047 vol.6.

[11] M. Spong, "The swing up control problem for the acrobot," IEEE Contr. Syst. Mag., vol. 15, pp. 49-55, 1995.

[12] C. Chung and J. Hauser, "Nonlinear control of a swinging pendulum," Automatica, vol. 31, no. 6, pp. 851-862, 1995.

[13] K. Åström and K. Furuta, "Swinging up a pendulum by energy control," Automatica, vol. 36, pp. 287-295, 2000.

[14] M. Yamakita, K. Nonaka, and K. Furuta, "Swing up control of a double pendulum," in Proc. ACC, 1993, pp. 2229-2233.

[15] N. Zoso and C. Gosselin, "Point-to-point motion planning of a parallel 3-dof underactuated cable-suspended robot," in Proc. IEEE ICRA, 2012, pp. 2325-2330.

[16] L. S. Cicolani and G. Kanning, "Equations of motion of slung load systems with results for dual lift," NASA Technical Memorandum 102246, Tech. Rep., 1990.

[17] P. Donner, A. Mörtl, S. Hirche, and M. Buss, "Human-robot cooperative object swinging," in Proc. IEEE ICRA, 2013, pp. 4343-4349.

[18] P. Donner, F. Christange, and M. Buss, "Human-robot cooperative swinging of complex pendulum-like objects," in Proc. IEEE IROS, Nov 2013, pp. 4602-4608.

[19] J. A. Acebrón, L. L. Bonilla, C. J. P. Vicente, F. Ritort, and R. Spigler, "The kuramoto model: A simple paradigm for synchronization phenomena," Reviews of modern physics, vol. 77, no. 1, p. 137, 2005. 